\title{
Sympatric Epichloë species and chemotypic profiles in natural populations of Lolium perenne
}

\author{
Milton C. Soto-Barajasª,b, Beatriz R. Vázquez-de-Aldanaa, Amador Álvarezª , Iñigo \\ Zabalgogeazcoa ${ }^{a}$ \\ anstitute of Natural Resources and Agrobiology of Salamanca. Consejo Superior de \\ Investigaciones Científicas (IRNASA-CSIC) \\ Cordel de Merinas 40-52, 37008 Salamanca, Spain \\ 'Instituto Tecnológico de Chiná, Calle 11 SN, 24520 Campeche (México). \\ Author for correspondence: Iñigo Zabalgogeazcoa \\ IRNASA-CSIC, Cordel de Merinas 40-52, 37008 Salamanca, Spain
}

i.zabalgo@irnasa.csic.es

Phone +34 923219606 


\section{ABSTRACT}

The distribution of different Epichloë species within eight natural populations of Lolium perenne was studied. In total, $40.2 \%$ of the asymptomatic plants were infected by Epichloë festucae var. Iolii or by Epichloë typhina. Both species occurred in sympatry at seven populations, and some plants had dual infections by both taxa. No hybrid taxa such as Epichloë hybrida were detected. Epichloë festucae strains were classified into two morphotypes, M1 and $M 3$, according to culture characters, both morphotypes occurred in sympatry at seven populations. Plants bearing stromata produced by Epichloë typhina were observed, but asymptomatic plants infected by this species also occurred at seven populations. The alkaloid profile of Lolium perenne plants was related to the morphotype of their infecting strains: Most plants infected by M3-strains were characterized by lolitrem, and those with M1-strains contained either ergovaline or lolitrem. Plants infected by Epichloë typhina were characterized by high peramine content.

Index descriptors: Epichloë, endophytes, alkaloids, grass, Lolium perenne, ergovaline, peramine, lolitrem. 


\section{INTRODUCTION}

The earliest known reports about fungal endophytes date from the late 19th century, when the presence of hyphae inside seeds of darnel (Lolium temulentum) was described. In one of those reports Guérin (1898) questioned how the fungus arrived to seeds, as well as its possible role in the toxicity of this plant species. Since then, fungal endophytes did not receive much attention until eight decades later, when endophytic Epichloë species were found to be responsible for the toxicity of grass pastures used for livestock grazing in the United States and New Zealand (Bacon et al., 1977; Fletcher and Harvey, 1981). Subsequent discoveries of toxic fungal alkaloids and improved performance of infected plants under some stress conditions, triggered research in Epichloë-grass associations (Clay and Schardl, 2002; Rodriguez et al., 2009; Saikkonen et al., 2016).

Leuchtmann et al. (2014) listed 43 taxa, including species and subspecies, within the genus Epichloë. Since then, some additions and nomenclatural changes have been made on that classification (i.e. Chen et al., 2015; Campbell et al., 2017; Shymanovich et al., 2017). All these taxa infect systemically the intercellular tissue of leaves and stems of particular grass hosts. Epichloë infections are chronic and usually asymptomatic in their host plants, and most species of this genus can be vertically transmitted to seed. An exception to this are some pathogenic species like E. typhina, which sterilize infected plants with fungal stromata that inhibit panicle development (choke disease), and it is not seed-transmitted in Lolium perenne, although in other host species seed transmission occurs (Tadych et al., 2014 ). Other Epichloë species display a mixed type lifestyle, usually being asymptomatic and seed transmitted, but occasionally producing stromata in one or a few stems in a small number of infected plants (i.e. Epichloë festucae) (Leuchtmann et al., 1994; Tadych et al., 2014).

Lolium perenne (perennial ryegrass; Fam. Poaceae), a perennial grass of eastern Mediterranean origin (Inda et al., 2014), is one of the most important agricultural species used for forage in temperate areas of the world, as well as for ornamental and sporting lawns. This grass is a host of the asymptomatic endophytes Epichloë festucae var. Iolii (= Neotyphodium Iolii) and Epichloë hybrida (= LpTG-2), an E. festucae var. Iolii x Epichloë typhina allopolyploid hybrid. In addition, L. perenne is a host of the pathogen E. typhina, causal agent of choke disease (Christensen et al., 1993; Schardl et al., 1994; Leuchtmann et al., 2014; Campbell et al., 2017). However, the pattern of occurrence of these species in natural populations of $L$. perenne is unknown.

Epichloë species are responsible for the synthesis of ergot alkaloids (ergovaline) and indole-diterpenes (lolitrem B, janthitrems) associated with mammalian toxicity, and peramine and lolines, with insecticide properties. These alkaloids enhance the competitive ability of 
endophyte-infected grasses protecting them from herbivory (Siegel and Bush, 1996; Bush et al., 1997; Schardl et al., 2013). In perennial ryegrass infected with E. festucae var. lolii, alkaloids of all classes except lolines have been detected. Besides the endophyte genetic background, alkaloid synthesis in plants is affected by factors like the host plant genotype, tissue, and phenological stage, as well as the surrounding environment (Lane et al., 2000).

There is great interest in the use of endophytes for the improvement of forage and turf cultivars of agricultural host grasses. For example, the persistence of forage cultivars of Lolium perenne or Schedonorus arundinaceus can be improved with Epichloë strains that do not produce alkaloids toxic to livestock, like lolitrem B or ergovaline (Bouton et al., 2002; Moate et al., 2012; Lugtenberg et al., 2016; Saikkonen et al., 2016). In contrast, the presence of those herbivore deterrent alkaloids can be advantageous to enhance the persistence of lawns and sports fields, or to reduce the presence of birds, mice and invertebrates in airports (Pennell et al., 2016). Variation in alkaloid production occuring among strains present in natural populations of grasses can be exploited to obtain strains useful for cultivar improvement (Vázquez de Aldana et al., 2010; Johnson et al., 2013; Young et al., 2013). Alternatively, alkaloid production pathways can be artificially disrupted in particular Epichloë strains (Florea et al., 2016; Mulinti et al., 2016).

Studies of the intrapopulation structure of Epichloë-grass associations in terms of genotypic, taxonomic, or chemical variation are rare, and to our knowledge, none is available for endophytes from natural populations of Lolium perenne. Christensen et al. (1991) observed variation in the appeareance of fungal cultures obtained from cultivar and ecotype collections of L. perenne. Bony et al. (2001) also reported phenotypic and species diversity among Epichloë cultures obtained from a collection of seeds from natural populations. Intrapopulation species or phenotypic variation was not determined in the above studies because only one or two endophytes per population were isolated. This background led us to set as a main objective to study the intrapopulation composition of natural stands of Lolium perenne in terms of their endophytes and alkaloid chemotypes.

\section{MATERIALS AND METHODS}

\section{Plant material}

In the spring of 2012, 344 asymptomatic plants of Lolium perenne and 14 with symptoms of choke disease (presence of Epichloë stromata in reproductive stems) were collected at eight populations with different ecological characteristics in western Spain (Table 1). In these wild populations, L. perenne plants were small and grew mixed with other plant species. Asymptomatic plants were collected randomly and symptomatic plants selectively. In 


\section{Isolation of Epichloë from plants}

Before the L. perenne plants were transplanted to the field plot, the presence of endophytes was diagnosed by means of fungal isolation. Samples of several reproductive stems or leaf sheaths from each plant were cut into pieces approximately $5 \mathrm{~mm}$ long, and surface sterilized for 10 minutes in a $20 \%$ commercial bleach solution ( $1 \%$ active chlorine), rinsed with sterile water, and placed into $9 \mathrm{~cm}$ Petri plates containing potato dextrose agar (PDA) with $200 \mathrm{mg} \mathrm{L}^{-1}$ of chloramphenicol. The plates were incubated in the dark at room temperature $\left(\approx 20-22{ }^{\circ} \mathrm{C}\right)$ and examined daily until endophytic mycelium emerged from infected plants. Then, a small amount of mycelium was transferred to a new PDA plate to obtain a culture. The presence of Epichloë was also confirmed by microscope examination of the stem pith, leaf sheaths, and seeds of perennial ryegrass stained with aniline blue (Bacon and White, 1994).

In some plants, both healthy reproductive stems and others bearing stromata occurred. In these cases, seeds produced in healthy stems were sown to check later for Epichloë infection in individual seedlings.

\section{Morphological classification of cultures}

A preliminary classification of the Epichloë cultures was based on their macro- and micromorphological characteristics. Macromorphological characters such as size, color, and surface texture of all the fungal cultures were observed on $9 \mathrm{~cm}$ Petri plates of three different culture media: PDA, rose Bengal chloramphenicol agar (RBA), and malt extract agar (MEA). 
Radial growth was measured for four weeks in 20 individual Epichloë strains in PDA, and the average daily growth rate was estimated based on four replicates of each culture. The presence and shape of conidia were analyzed in cultures of PDA and water agar maintained at room temperature and at $10^{\circ} \mathrm{C}$, which is a requirement for the sporulation of E. festucae var. lolii (Christensen et al., 1991). To do this, small blocks $(0.5 \times 0.5 \mathrm{~cm})$ of culture medium were removed from the margins of colonies, observed at the microscope with a cover slip placed on top of the block, and photographed to analyze the shape and size of conidia $(n=20)$.

\section{Genotypic classification}

A genotypic classification of the endophytes was based on the nucleotide sequence of the ITS1-5.8S rDNA-ITS2 region, and a 5 ' region of the $\beta$-tubulin (tubB) gene. The oligonucleotide pairs ITS4 / ITS5 (White et al., 1990), and tub2-exon1d-1 / tub2-exon4u-2 (Moon et al., 2002) were used to amplify each one of these regions. Epichloë DNA was extracted from a small amount of mycelium scraped from PDA cultures using a commercial kit (RedExtract-n-Amp, Sigma-Aldrich). For both DNA targets the PCR assay had the same conditions: 2 minutes at $95^{\circ} \mathrm{C}, 35$ cycles of 1 min at $94{ }^{\circ} \mathrm{C}, 1 \mathrm{~min}$ at $54^{\circ} \mathrm{C}$ and $1 \mathrm{~min}$ at $72{ }^{\circ} \mathrm{C}$; and a final extension of $10 \mathrm{~min}$ at $72{ }^{\circ} \mathrm{C}$.

Both strands of each replicon were sequenced in a 3100 Genetic Analyzer (Applied Biosciences). Sequence chromatograms were analyzed with Chromas LITE 2.1.1 software (Technelysium Pty Ltd). To analyze the grouping of distinct genotypes, reference sequences of known Epichloë strains were used, and phylogenetic trees were constructed using the maximum likelihood method. Evolutionary distances were computed using the Tamura 3parameter method. Robustness of the genotypic classifications was estimated by bootstrapping using MEGA version 6 software (Tamura et al., 2013). Initial trees were made with all strain sequences available, afterwards, for better visualization condensed trees were made with one sequence representative of each group of identical sequences of strains from each location and morphotype.

The E. hybrida strain AR6 (E. typhina x E. festucae var. lolii), donated by Dr. Linda Johnson (AgResearch, New Zealand), was used as a hybrid species of reference. To detect Epichloë hybrids among the strains obtained, all the tubB sequences were checked for presence of overlapping peaks in chromatograms, indicating that an isolate might have two alleles of this gene. Additionally, hybrid condition was evaluated by a PCR amplicon cloning procedure. Amplicons of the tubB gene of several Epichloë strains representative of each morphotype, including the AR6 hybrid, were inserted into pJET1.2/blunt plasmids (Thermo Scientific), and used to transform competent Escherichia coli DH5a cells. Six colonies of the 
transformed E. coli, containing the Epichloë tubB inserts of each morphological group were sequenced to compare the inserts. The AR6 hybrid is a heteroploid containing two alleles of the tubB gene, therefore, sequence differences between tubB inserts carried by different $E$. coli transformants would be expected to occur with this strain (Moon et al., 2004).

\section{Alkaloid analyses}

The concentration of peramine, lolitrem B and ergovaline alkaloids was determined in 119 plants previously diagnosed by fungal isolation as infected by a single endophyte (96 plants), double infected (8), or endophyte free (15). These plants, originally from CR, LVA, LVE, POR, TAB and VAF locations, were harvested from the field plot at the flowering stage on May 2014. Whole plants, including leaves and reproductive stems were cut $5 \mathrm{~cm}$ above the soil line, stored at $-80^{\circ} \mathrm{C}$, freeze-dried, and ground to $0.5 \mathrm{~mm}$ with a hammer mill (Fritsch 15303). Each alkaloid was analyzed separately, using some modifications of published methods based on high-performance liquid chromatography (HPLC).

Peramine was extracted according to Barker et al. (1993) and quantified by comparing samples with a standard donated by G. Lane (AgResearch, New Zealand). The analysis was performed by HPLC (Waters module 2695) with a C18 column 150 x 3.9 mm, $4.0 \mu \mathrm{m}$ (Waters Nova Pak 036975) using a Photodiode Array detector (Waters 996) set at $230 \mathrm{~nm}$. The mobile phase was isocratic, composed of $15 \%$ acetonitrile, $85 \%$ of $10 \mathrm{mM}$ guanidine carbonate buffer, and $0.16 \%$ formic acid, with a flow rate of $0.7 \mathrm{~mL} \mathrm{~min}^{-1}$. The peramine peak was detected at $3.5 \min$

Quantification of lolitrem B was based on Gallagher et al. (1985), using a standard provided by C. Miller (AgResearch, New Zealand) in a HPLC with a Waters 2695 module, a silica column 250 x $4.6 \mathrm{~mm}, 5.0 \mu \mathrm{m}$ (Waters Spherisorb) and a fluorescence detector (Waters 2475) $\lambda_{\mathrm{exc}}=268 \mathrm{~nm} ; \lambda_{\mathrm{em}}=440 \mathrm{~nm}$. The mobile phase was composed of $80 \%$ dichloromethane and $20 \%$ acetonitrile, with flow rate of $1.0 \mathrm{~mL} \mathrm{~min}{ }^{-1}$. The retention time for lolitrem $\mathrm{B}$ was $5.9 \mathrm{~min}$.

The procedure described by Yue et al. (2000) was used to determine the concentration of ergovaline with an internal standard of ergotamine (Sigma-Aldrich). Quantification was by reverse phase HPLC in a Waters 2695 module, a C18 column $150 \times 4.6 \mathrm{~mm} ; 2.7 \mu \mathrm{m}$ (Agilent Poroshell) and a fluorescence detector (Waters 2475) $\lambda_{\mathrm{exc}}=250 \mathrm{~nm} ; \lambda_{\mathrm{em}}=420 \mathrm{~nm}$. The initial solvent was $35 \%$ acetonitrile in $0.01 \mathrm{M}$ ammonium acetate, with a flow rate of $0.8 \mathrm{~mL} \mathrm{~min}{ }^{-1}$. The gradient was adjusted through time programming as follows: step 1, 35\% to $50 \%$ acetonitrile in a 20 min linear gradient; step 2, 50\% for $5 \mathrm{~min}$; step 3, 50\% to $90 \%$ in a $5 \mathrm{~min}$ linear gradient; step $4,90 \%$ to $35 \%$ in a 5 min linear gradient. The ergovaline standard was purchased from F. Smith (Auburn University, USA), and it was detected at $13.3 \mathrm{~min}$. 
The percentage of plants that produced peramine, lolitrem B and ergovaline was calculated for the complete set of samples presumably infected by a single endophyte. The concentration of each alkaloid in plants was compared across the morphotypes (M1, M2, M3) by means of a one way ANOVA followed by a post-hoc Bonferroni test. These statistical analyses included only L. perenne plants infected with a single endophyte (dual infections were considered separately) and producing detectable amounts of the alkaloid (zeros were not included).

To search for possible relationships among different fungal morphotypes, and the alkaloid content (peramine, lolitrem B and ergovaline) of their host plants, a Principal Component Analysis (PCA) was run with a matrix of 104 rows (samples) considering the concentration of the alkaloids as variables. For the statistical analyses SPSS 23.0 software (SPSS Inc., Chicago, IL, USA) was used.

\section{RESULTS}

\section{Epichloë prevalence and morphotype classification}

The presence of endophytic Epichloë was detected in 140 of the 344 asymptomatic L. perenne plants which were sampled and diagnosed. The mean prevalence of Epichloë-infected plants $(E+)$ was of $40.7 \%$, ranging from $32.1 \%$ in VAF to $54.6 \%$ in CR (Table 1). The 14 plants showing choke disease symptoms at sampling time were not included in the calculations of prevalence, because they were chosen selectively, rather than randomly. The Epichloë cultures obtained from asymptomatic plants displayed different macromorphological characteristics, but major similarities and differences among them allowed their classification into three distinct morphotypes (Figure 1): (i) M1 morphotype, hyphae of this group of fungi emerged from plant tissues about one month after placing ryegrass samples on PDA. Cultures had strongly aggregated 'brain-like' white mycelium and the slowest growth rate $(\bar{x}=0.19 \pm 0.02 \mathrm{~mm}$ day $^{-1}$ ) of all morphotypes. (ii) M2 morphotype, hyphae emerged about five days after the ryegrass samples were placed in PDA plates. Cultures had white color with abundant cottony aerial mycelium and the fastest growth rate $\left(\bar{x}=1.54 \pm 0.16 \mathrm{~mm}\right.$ day $\left.{ }^{-1}\right)$. (iii) $\mathrm{M} 3$ morphotype, whose hyphae emerged from plant fragments after about two weeks, had flat and smooth mycelium of light tan color and a growth rate of $0.47 \pm 0.06 \mathrm{~mm} \mathrm{day}^{-1}$. All endophytes isolated from plant stems that bore Epichloë stromata (choke disease) (Figure S1) had cultures with M2 morphotype characteristics, and were designated M2S.

Conidia were observed in cultures of the M1 and M2 morphotypes, and of the $E$. hybrida AR6 strain, although different conditions were required for their production. The cultures of the M1 morphotype produced conidia only in water agar and after three weeks of 
incubation at $10^{\circ} \mathrm{C}$, whereas those of the $\mathrm{M} 2$ morphotype and $\mathrm{E}$. hybrida produced conidia at room temperature in PDA (Figure 1). However, cultures of the M3 morphotype were sterile in all media and temperatures tested. The conidia of the M1 and M2 morphotypes and the AR6 strain had the reniform shape characteristic of the genus Epichloë, but with statistical differences in size $(P<0.001)$ : conidia of $M 1$ and $M 2$ morphotypes had similar length, $4.96 \pm 0.18$ $\mu \mathrm{m}$ and $5.07 \pm 0.09 \mu \mathrm{m}$ respectively; whereas the AR6 hybrid produced larger conidia of $7.80 \pm 0.44 \mu \mathrm{m}$. The observed length of the AR6 conidia is similar to that of other hybrid species like $E$. coenophiala, while that of the $M 1$ and $M 2$ strains resembled the length of non-hybrid Epichloë species (Schardl and Leuchtmann, 2005).

\section{Morphotype distribution in locations and plants}

In asymptomatic plants different Epichloë morphotypes occurred in sympatry at several locations (Table 1). The most common endophytes belonged to the M1 morphotype, $47.3 \%$ of the cultures obtained had this morphotype. Endophytes with the M2 morphotype represented $13.3 \%$ of the cultures, and the remaining $39.3 \%$ of the cultures were of M3 morphotype (Table 1). In addition, 14 cultures with M2 morphotype were obtained from an equal number of plants showing stromata at the time they were sampled in the field, and these cultures were referred as M2S to distinguish them from cultures of similar morphotype obtained from asymptomatic plants.

At six locations (all except LVE and PI) the three Epichloë morphotypes (M1, M2, M3) occurred in sympatry, and in three of these locations (CR, LVA and POR) plants with choke disease and M2S strains occurred. Plants from LVE, the only sampling site adjacent to agricultural lands, had the most homogeneous endophyte population, except for a single plant harboring an M2 endophyte all other cultures belonged to the M1 morphotype. The only location where no M2 morphotypes were isolated was PI (Table 1).

From 140 asymptomatic E+ plants, 150 Epichloë cultures were obtained; this happened because from 10 of these plants two endophytes of different morphotypes were isolated (Table 1). In addition, five of the 14 plants which originally had stromata also had double infections. These five plants produced both healthy reproductive stems with seed heads, and stems with stromata one year after the transplant to the field plot. From each of these plants $\mathrm{M} 2 \mathrm{~S}$ endophytes were isolated from choked stems, and ten seeds were collected from healthy stems, germinated, and transplanted to pots. Three months later, Epichloë seed transmission was verified by isolation, as M1 and M3 endophyte cultures were obtained from seedlings. Therefore, two different Epichloë morphotypes were isolated from different stems of these 
five plants: three plants from LVA and one from POR were infected by M2S and M1 endophytes, and one plant from CR with M2S and M3 endophytes.

\section{Taxonomic classification}

The first genotypic classification of Epichloë strains was based on 86 ITS sequences of about $480 \mathrm{bp}$ of length obtained from cultures representing all the Epichloë morphotypes and locations, plus reference sequences (Supplementary Fig. S2). A phylogenetic analysis clustered all genotypes in two major clades (Fig. 2), one constituted by M1 and M3 morphotypes and reference sequences of $E$. festucae var. lolii, and another constituted by M2 and M2S morphotypes and reference sequences of E. typhina. The first clade was composed by three groups, differentiated among them by single nucleotide substitutions; this difference was corroborated by resequencing some samples of each subclade at least two times. The first group contained identical sequences of M1 and M3 strains from various locations. Another group was defined by a sequence common to $20 \mathrm{M} 1$ strains isolated only from the LVE and VAF locations. A third group was observed in four sequences belonging to M1 strains isolated from plants from DIV, PI and POR. A unique sequence was detected in the LVA27 isolate, an M3 morphotype. The strains of E. festucae var. Iolii were grouped in a clade different from $E$. festucae strains isolated from Festuca rubra. A second major clade contained identical sequences of the M2 and M2S morphotypes, only the sequence of the CR16 isolate, an M2 morphotype, was different from the others.

Another phylogenetic analysis was made with partial tubB gene sequences from 80 strains (Supplementary Fig. S3). It was based on an 836 nucleotide long sequence alignment, with 53 variant sites (excluding deletions), 37 of which were informative. Like the rDNA dendrogram, the tubB dendrogram grouped the strains into two main clades corresponding to E. festucae and E. typhina (Fig. 3). There were no differences among the M1 and M3 morphotypes. On the other hand, the E. typhina clade was divided into two groups because of one variant site. One group contained both asymptomatic and stromata forming isolates, and the other consisted of four stromata-forming endophytes.

The correlation between morphotype and taxon was highly consistent. Using either ITS or tubB sequences, all M1 and M3 strains sequenced grouped into a E. festucae clade, and all M2 and M2S strains sequenced grouped into a E. typhina clade. No exceptions from this occurred.

To find out if an E. typhina strain from a different host grass could have infected $L$. perenne, and be the source of one of the two groups observed in the tubB dendrogram, reference sequences of E. typhina strains from different grass species (Leuchtmann et al., 
2014) were compared to those two genotypes (Fig. 4). One genotype was identical to reference sequences of $E$. typhina from $L$. perenne, and these were the most similar to the other genotype in the complete set of sequences representative of E. typhina strains from different hosts. The strains from Holcus lanatus were the most closely related to those of Lolium perenne, but differed in sequence from the two Epichloë genotypes obtained from $L$. perenne. Therefore, a recent event of cross species inoculation seems to be an unlikely origin of any of these E. typhina genotypes detected in L. perenne.

The two tubB sequences of the E. hybrida AR6 strain, identified as AR6-a and AR6-b, were allocated to different clades (Fig. 3). The AR6-a sequence differed in one nucleotide from reference sequences of E. festucae var. Iolii, and the AR6-b sequence had a single substitution with respect to the reference sequence of $E$. typhina.

Epichloë hybrids were not detected through cloning of PCR amplicons. The six sequences of the DH5a cells transformed with tubB amplicons from strains belonging to the M1, M2, M2S and M3 morphotypes were 100\% identical. On the other hand, some sequences of the transformants from the AR6 hybrid, were almost identical to a reference sequence of $E$. festucae var. Iolii and the other sequences to E. typhina (Fig. 3).

In none of the tubB sequence chromatograms of the Epichloë isolates ambiguous peaks suggesting hybrid constitution were observed (Schardl et al., 1994). However, in the chromatograms of the AR6 hybrid sequence, overlapping peaks were observed at positions where the tubB copies of the parental E. festucae var. Iolii and E. typhina differed in nucleotide sequence (Supplementary Fig. S1). This observation suggests that the hybrid or non-hybrid constitution of an endophyte can be easily discerned in tubB sequence chromatograms.

\section{Fungal alkaloids in infected plants}

The presence of at least one of the three alkaloids analyzed (peramine, lolitrem B, ergovaline) was detected in 94 of the $104 \mathrm{E}+\mathrm{L}$. perenne samples analyzed. A preliminary statistical analysis showed that variation in alkaloid concentration across plant locations was mainly due to the morphotype of the fungus. Therefore, the statistical analysis of alkaloid content in plants was focused on the effect of the Epichloë morphotype.

Peramine was the most frequent alkaloid in the L. perenne plants, it was detected in $70.8 \%$ of the 96 single infected samples, and in all double infections (Table 2). The lowest peramine concentration $(\mathrm{P}<0.001)$ was detected in plants infected with the M1 morphotype, and M2S-infected plants had the highest concentrations.

Lolitrem B was detected in $61.5 \%$ of the 96 single infected samples, and in six of the eight double infected. Plants with M3 endophytes had a significantly higher concentration of 

$\mathrm{kg}^{-1}$, the threshold for causing ryegrass staggers in cattle and sheep (di Menna et al., 1992), were detected in five M3 and two M1 plants.

Ergovaline was found in $54.2 \%$ of the single infected samples, and in three of the eight double infected. It was the least common of the three alkaloids, although it was present in $84.6 \%$ of the $M 3$ infected plants. Plants with stromata did not contain this alkaloid, and differences in concentration among plants with $M 1, M 2$ and $M 3$ morphotypes were not statistically significant (Table 2). Most plants containing ergovaline had a concentration above $0.4 \mathrm{mg} \mathrm{kg}^{-1}$, a content associated with increased sensitivity to heat stress in cattle fed on such forage (Hovermale and Craig, 2001).

The presence of the three alkaloids -peramine, lolitrem B and ergovaline- was detected in 19 out of 26 plants with M3 morphotype, in 9 out of 53 plants with M1, in one out of 8 plants with $\mathrm{M} 2$, and in no plant with M2S. The only plants containing peramine and no ergovaline nor lolitrem had stromata produced by E. typhina. One plant with M2 morphotype (CR13) was found to have high peramine $\left(20 \mathrm{mg} \mathrm{kg}^{-1}\right)$, no ergovaline and low lolitrem B $(0.5 \mathrm{mg}$ $\left.\mathrm{kg}^{-1}\right)$

All eight double infected (DI) plants contained peramine, six had lolitrem B, and three ergovaline (Table 2). In the asymptomatic M2/M1 plants the three alkaloids were detected, and they had the lowest peramine content; plants with M2/M3 endophytes contained lolitrem $B$ and high peramine levels but no ergovaline. Double infected plants with stromata (M2S/M1, $\mathrm{M} 2 \mathrm{~S} / \mathrm{M} 3$ ) had high levels of peramine, did not contain ergovaline and one plant (M2S/M1) had lolitrem B.

\section{Relationship between fungal morphotypes and alkaloid content}

A principal components analysis (PCA) showed interesting relationships between alkaloid content in plants and the morphotype of fungal strains. The first two principal components accounted for $73.6 \%$ of the total variance $(\mathrm{PCI}=41.6 \%$ and $\mathrm{PCII}=32.1 \%)$. The component I was characterized by lolitrem B in the positive part (0.692) and by ergovaline in the negative part $(-0.751)$, and the component II by peramine in the positive part $(0.870)$ and lolitrem $B$ in the negative $(-0.436)$.

In the PCA classification two major groups were clearly distinguished (Fig. 5). A first group along component I was related to lolitrem B and ergovaline contents and consisted mainly of plants with M1 and M3 morphotypes. Within this group, two subgroups were differentiated: one related to lolitrem B consisting of most plant samples with $\mathrm{M} 3$ morphotype (producing the three alkaloids), together with M1 morphotypes with no ergovaline and 
variable peramine contents (mainly from LVE and VAF locations). The second subgroup was related to ergovaline content and consisted mostly of M1-plants with no lolitrem B and variable peramine levels; few samples with morphotypes $M 2$ and $M 3$ were also in this subgroup. Close to this subgroup ten samples with different morphotypes (M1, M2, M3 and M2S) were clustered together and characterized by not producing any alkaloid. The second main group was related to peramine and included most plants infected by E. typhina and with dual infections (Fig. 5). Within this group, two different subgroups were differentiated along the bisector of the quadrant: samples containing lolitrem B were located below this line and samples without lolitrem B were above.

\section{DISCUSSION}

Endophytes were present at all the locations studied, and on average $40.7 \%$ of the asymptomatic plants of Lolium perenne were infected. This prevalence is high in comparison to those observed in other European surveys, with mean values ranging from 6\% to 33\% (Lewis et al. 1997; Oliveira and Castro, 1998; Jensen and Roulund, 2004; Dobrindt et al., 2013; König et al., 2018). Greater prevalence of Epichloë infections has been reported in warm and dry areas, suggesting that such conditions may impart a selection pressure that favors Lolium-Epichloë symbioses (Lewis et al., 1997; Hesse et al., 2003; Gibert et al., 2012). However, the location with the highest precipitation in this survey ( $\mathrm{Pl}$, mountain pasture with $780 \mathrm{~mm} \mathrm{yr}^{-1}$ ) was not the one with the lowest prevalence, suggesting that other factors also affect the maintenance of endophyte symbioses.

Like previous studies, we observed significant phenotypic variation among Epichlöe cultures obtained from L. perenne (Christensen et al., 1991; Bony et al., 2001). After careful observation of phenotypic characters such as culture growth rate, color, morphology, and the presence or absence of conidia, 150 isolates were classified into three distinct morphotypes (M1, M2, M3). According to their ITS and tubB nucleotide sequences, all M1 and M3 isolates belonged to E. festucae var. lolii, and both morphotypes fit descriptions given for this taxon (Christensen et al., 1991; Bony et al., 2001). All M2 isolates, including those obtained from plants with stromata (M2S), were identified as E. typhina.

The results obtained in this study provide new information about the structure of natural populations of Epichloë in Lolium perenne. Two different Epichloë species, E. festucae var. Iolii and E. typhina occurred in sympatry in wild populations of $L$. perenne. These two taxa coexisted at seven of the eight populations analyzed, and both M1 and M3 strains of $E$. festucae var. Iolii also occurred in sympatry at seven of the eight populations. In addition, 7.1 $\%$ of the asymptomatic plants analyzed were dually infected by strains belonging to both 
Epichloë species. Possibly this figure is an underestimation of the actual frequency of double infections, as explained later. Christensen et al. (2000) showed that dual infections can be established in L. perenne seedlings after artificial inoculation. The results of our study show that dual infections occur in natural populations.

The existence of E. festucae var. Iolii and E. typhina in sympatry, plus dual infections by these taxa in ryegrass plants, confirm the scenario predicted by Schardl et al. (1994) where anastomosis and karyogamy of E. festucae var. lolii and E. typhina in double infected plants could lead to the generation of $E$. hybrida. However, none of the isolates analyzed seemed to be a hybrid, as deduced from sequence chromatograms, amplicon cloning, or spore size. This absence of hybrids in populations where potential ancestral species are present contrasts with Epichloë-Hordelymus europaeus associations, where a high prevalence of hybrids has been found in populations where several Epichloë taxa occur (Oberhofer and Leuchtmann, 2012). Somatic hybridization might not be a straightforward process in Epichloë, because attempts to obtain hybrids by means of multiple artificial inoculations in Hordelymus europaeus have failed (Oberhofer and Leuchtmann, 2014). Environmental conditions different from those of the populations we studied might facilitate hybrid generation. Epichloë hybrida has been identified in southern France (Campbell et al., 2017), perhaps these French populations might contain the parental taxa, as in Spain, in addition to E. hybrida.

The double infections observed always consisted of an E. festucae var. lolii M1 or M3 strain, together with an E. typhina strain; double infections by $M 1$ and $M 3$ strains were not observed. This could be explained because transmission of E. festucae var. Iolii is exclusively vertical to seeds (Tadych et al., 2014), but developing seeds infected by E. festucae could be horizontally superinfected by E. typhina ascospores discharged from stromata. Such an event was proposed by Schardl et al. (1994) as a first step for the generation of E. hybrida. A similar situation might occur in Brachypodium sylvaticum, where multiple infections by asymptomatic and stroma forming strains of Epichloë sylvatica were observed in natural populations (Meijer and Leuchtmann, 1999).

In our study, double infections in asymptomatic plants were detected by plating together several reproductive stems and/or leaf sheaths from each plant. Therefore, we do not know if both fungal strains occurred in the same or in different tillers. Further work on the distribution of Epichloë strains in double infected plants of Lolium perenne is a future aim of our research. Concerning strain distribution in multiple infections of single plants, studies of natural infections in Brachypodium sylvaticum and artificial inoculations in L. perenne and Bromus erectus suggest that in double infected plants different strains tend to be separated in different tillers (Meijer and Leuchtmann, 1999; Wille et al., 1999; Christensen et al., 2000). In 
these studies different fungal strains were first isolated from single plants and later identified. More recently Oberhofer and Leuchtmann (2014) diagnosed multiple infections using DNA extracted from tillers of artificially inoculated plants, and in this case multistrain infections in the same tiller were relatively common. It is possible that the results of studies where fungal isolation was used were technically limited to detect double infections in the same tiller because of different growth rates of different Epichloë taxa or strains in isolation medium. If we would have used a single tiller isolation method to determine strain distribution in double infected plants, we might have missed $E$. festucae strains, particularly M1 type, because $E$. typhina grows much faster in isolation medium. On the other hand, if we would have used single tiller DNA extracts for this purpose, we might have missed the discrimination of $E$. festucae into M1 and M3 morphotypes. Therefore, both isolation and DNA techniques should be used in future studies of the distribution of strains in single plants.

Although rDNA and tubB genotypes suggested that $M 1$ and $M 3$ isolates belong to $E$. festucae var. Iolii, the phenotypic distinction between cultures of both morphotypes is evident. In addition, a discrimination between plants infected by $\mathrm{M} 1$ and $\mathrm{M} 3$ morphotypes was possible by means of near infrared reflectance spectroscopy (NIRS) (Soto-Barajas et al., 2018). Whether M1 and M3 isolates belong to different species is a question that deserves further research. Recently, a new Epichloë taxon tentatively named LpTG-3 has been proposed (Hettiarachchige et al., 2015). The description of this non-hybrid taxon is based on a small number of strains isolated from Lolium perenne in France, and its tubB genotype is similar to that of E. festucae var. Iolii. However, plants infected by LpTG-3 are devoid of lolitrem B, ergovaline and peramine (Kaur et al., 2015). The M3 strains are not likely to belong to this taxon because out of 26 plants analyzed, only three did not have any of these alkaloids, 19 had the three alkaloids, three had ergovaline and peramine, and one had lolitrem B.

The chemotypic profiles of infected plants were related to culture morphotypes. According to PCA, there was a relationship between the fungal morphotype and the type of alkaloids contained in the host plant. Some plants infected with the M1 morphotype had the highest ergovaline content of all plants, those infected with M3 strains contained high levels of lolitrem $B$; and the highest amounts of peramine were detected in plants infected with $E$. typhina (M2S and M2 morphotypes). Bony et al., (2001) observed a close link between the deficiency of ergovaline or lolitrem in E. festucae var lolii and the morphology of isolates, and suggested that the 'mutation' (in the broad sense) which lead to the absence of synthesis of lolitrem or egovaline is regularly accompanied by disturbances of the mycelial growth.

A surprising result was the fact that lolitrem B was detected in two plants with stromata, and four asymptomatic plants infected by M2 morphotypes. This could be due to 
undetected dual infections because only some, or none of the 11 genes of the indole diterpene (IDT) biosynthesis cluster, responsible for the synthesis of lolitrem B are present in the genomes of E. typhina strains analyzed so far (Schardl et al., 2013; Young et al., 2009). If this were general for E. typhina, plants infected by this fungus should not contain lolitrem B. Furthermore, lolitrem B was detected in $75 \%$ of the plants doubly infected with $E$. typhina and E. festucae var. Iolii (Table 2). However, a plant of L. perenne cv. Romance, artificially inoculated with M2 strain LVA32, and plants of L. perenne cv. Barplus, artificially inoculated with M2 strain LVA8 and M2S strain MON7, did not produce lolitrem B (Soto Barajas, 2016). This suggests that plants infected by E. typhina strains from our study do not contain lolitrem, and the two choked plants plus the asymptomatic M2 plants containing lolitrem could have been superinfected with E. festucae. Misclassification of some plants as individually infected instead of dually infected could have occurred because direct isolation in PDA was the method used to detect the presence of Epichloë in plants. In this process, E. typhina (M2 and M2S) strains grow much faster than E. festucae var. lolii (M1, M3), and this could have led to erroneous diagnosis of some double infected plants as only infected by E. typhina.

It is an interesting finding that E. typhina, considered a pathogen causing choke disease in all or most stems of host plants (Chung and Schardl, 1997), was isolated from $12.7 \%$ of the asymptomatic plants. Therefore, in its association with L. perenne, E. typhina can behave as an asymptomatic endophyte or as a pathogen. Some Epichloë species like E. festucae behave in this fashion when associated to Festuca rubra, being asyptomatic in most infected hosts, but occasionally forming stromata (Leuchtmann et al., 1994; Zabalgogeazcoa et al., 1999). However, Epichloë species with such mixed type life strategy are vertically transmitted by seed. Seed transmission of E. typhina has never been observed in L. perenne, but occurs in Puccinella distans and some plant species infected by E. typhina subsp. poae (Tadych et al. 2014). Therefore, another question that remains to be answered is whether seeds produced in asymptomatic L. perenne plants infected by E. typhina are carriers of this fungus. According to current knowledge they should not be. Environmental conditions such as nitrogen fertilization have been reported to affect the expression of stromata in E. festucae (Sun et al., 1990). In our study an environmental influence on stroma formation seems evident because some originally asymptomatic M2 plants produced stromata the year following field sampling, when they were transplanted to a field-plot.

In some of the L. perenne sampling sites other hosts of E. typhina such as Holcus lanatus and Dactylis glomerata occurred. The fact that the E. typhina clade formed by M2 isolates contained two distinct tubB genotypes suggested that infections by $E$. typhina from other grass species could have occurred. However, this seems unlikely, because the two $E$. 

Although E. typhina has been reported in several host grasses, this result supports that it is not a generalist species, but a complex of different taxa showing host specialization (Schirrmann and Leuchtmann, 2015).

The concentration of alkaloids in infected ryegrass was in the same range found by other researchers: peramine $2.0-52.8 \mathrm{mg} \mathrm{kg}^{-1}$, lolitrem B $0.3-11.5 \mathrm{mg} \mathrm{kg}^{-1}$, and ergovaline 0.24-3.46 mg kg-1 (Rowan et al., 1990; Christensen et al., 1991; Lane et al., 1997; Bony et al., 2001; Reed et al., 2004). Toxic levels of lolitrem B, with the potential to cause ryegrass staggers in cattle and sheep (>1.80 $\mathrm{g} \mathrm{kg}^{-1}$ ) (di Menna et al., 1992) were detected in a small number of plants, mostly among those infected with $\mathrm{M} 3$ endophytes. However, close to $90 \%$ of the samples contained ergovaline above the reported safe limit for livestock consumption $(0.40 \mathrm{~g}$ $\mathrm{kg}^{-1}$ ) (Hovermale and Craig, 2001). In spite of these results, we are not aware of any report concerning animal toxicity in Spanish pastures. Endophyte toxicosis is an important issue in monoculture or Lolium-dominated artificial pastures in New Zealand (Easton et al. 2001). However, endophyte toxicosis is rare in Europe because of the botanical diversity of natural pastures widely used for animal grazing, and the use of endophyte-free ryegrass cultivars (Zabalgogeazcoa and Bony, 2004).

In summary, several Epichloë taxa were previously known to infect Lolium perenne, and our work shows that two of these taxa, E. festucae var. lolii and E. typhina, commonly occur in sympatry in natural populations. Plants infected by both species also occur in these populations. This is an scenario expected to facilitate the generation of taxa such as E. hybrida, an E. festuae var. Iolii x E. typhina allopoliploid hybrid. However, we did not find hybrid strains in the populations analyzed. Two distinct morphotypes of E. festucae var. lolii were characterized according to culture macro and microscopic characteristics. These two morphotypes, M1 and M3, also occurred in sympatry at most populations analyzed, but no single plants infected by both morphotypes were found. Whether these to morphotypes represent two different taxa is not yet known. According to a PCA, the alkaloid profiles of host plants were related to the morphotype of the Epichloë strains that infect them. This study shows that intrapopulation surveys can reveal important information about the structure of natural populations of Epichloë species.

\section{ACKNOWLEDGMENTS}

This work was financed by research grants AGL2011-22783 and AGL2016-76035-C2-1R, funded by the Spanish Ministry of Economy and Competitivity and cofounded by FEDER. Dr. 
Soto-Barajas is a researcher from the Cátedras CONACYT program of the Mexican Council of Science and Technology. Thanks are due to Javier Gomez Fuertes for technical help.

\section{REFERENCES}

Bacon, C.W., Porter, J.K., Robbins, J.D., Luttrell, E.S. 1977. Epichloë typhina from toxic tall fescue grasses. Appl. Environ. Microbiol. 34, 576-581.

Bacon, C.W., White, J.F. 1994. Stains, media and procedures for analyzing endophytes, in Bacon, C.W., White, J.F. (eds), Biotechnology of Endophytic Fungi of Grasses. CRC Press, Boca Raton, FL, pp. 47-56.

Barker, D., Davies, E., Lane, G., Tapper, B.A. 1993. Effect of water deficit on alkaloid concentrations in perennial ryegrass endophyte associations, in Proceedings of the Second International Symposium on Acremonium/Grass Interactions. Palmerston North, New Zealand, pp. 67-71.

Bony, S., Pichon, N., Ravel, C., Durix, A., Balfourier F, Guillaumin JJ. 2001. The relationship between mycotoxin synthesis and isolate morphology in fungal endophytes of Lolium perenne. New Phytol. 152, 125-137.

Bouton, J.H., Latch, G.C.M., Hill, N.S., Hoveland, C.S., McCann, M.A., Watson, R.A., Parish, J.A., Hawkins, L.L., Thopson, F.N. 2002. Reinfection of tall fescue cultivars with non-ergot alkaloid-producing endophytes. Agron. J. 94, 567-574.

Bush, L.P., Wilkinson, H.H., Schardl, C.L. 1997. Bioprotective alkaloids of grass-fungal endophyte symbioses. Plant Physiol. 114, 1-7.

Campbell, M.A., Tapper, B.A., Simpson, W.R, Johnson, R.D., Mace, W., Ram, A., Lukito, Y., Dupont, P.Y., Johnson, L.J., Scott, D.B., Ganley, A.R.D., Cox, M.P. 2017. Epichloë hybrida, sp. nov., an emerging model system for investigating fungal allopolyploidy. Mycologia 109, 715-729.

Chen, L., Li, X., Li, C., Swoboda, G.A., Young, C.A., Sugawara, K., Leuchtmann, A., Schardl, C.L. 2015. Two distinct Epichloë species symbiotic with Achnatherum inebrians, drunken horse grass. Mycologia 107, 863-873.

Christensen, M.J., Latch, G.C., Tapper, B.A. 1991. Variation within isolates of Acremonium endophytes from perennial rye-grasses. Mycol. Res. 95, 918-923.

Christensen, M.J., Leuchtmann, A., Rowan, D.D., Tapper, B.A. 1993. Taxonomy of Acremonium endophytes of tall fescue (Festuca arundinacea), meadow fescue ( $F$. pratensis) and perennial raygrass (Lolium perenne). Mycol. Res. 97, 1083-1092.

Christensen, M.J., Simpson, W.R. Al Samarrai, T. 2000. Infection of tall fescue and perennial ryegrass plants by combinations of different Neotyphodium endophytes Mycol. Res. 104, 974-978.

Chung, K.R., Schardl, C.L. 1997. Sexual cycle and horizontal transmission of the grass symbiont Epichloë typhina. Mycol. Res. 101, 295-301.

Clay, K., Schardl, C.L. 2002. Evolutionary origin and ecological consequences of endophyte symbiosis with grasses. Amer. Nat. 160, 99-127.

Di Menna, M., Mortimer, P., Prestidge, R., Hawkes, A., Sprosen, J. 1992. Lolitrem B concentrations, counts of Acremonium lolii hyphae, and the incidence of ryegrass staggers 
in lambs on plots of A. lolii-infected perennial ryegrass. New Zealand J. Agric. Res. 35, 211217.

Dobrindt, L., Stroh, H.G., Isselstein, J., Vidal, S. 2013. Infected-not infected: Factors influencing the abundance of the endophyte Neotyphodium lolii in managed grasslands. Agric. Ecosyst. Environ. 175, 54-59.

Easton, H.S., Christensen, M.J., Eerens, J.P.J., Fletcher, L.R., Hume, D.E., Keogh, R.G., Lane, G.A., Latch, G.C.M., Pennell, C.G.L., Popay, A.J., Rolston, M.P., Sutherland, B.L., Tapper, B.A. 2001. Ryegrass endophyte: a New Zealand Grassland success story. Proc. New Zealand Grassland Assoc. 63, 37-46.

FAO/UNESCO 1998. World reference base for soil resources. In: World soil resources reports. FAO/UNESCO, Rome.

Fletcher, L.R., Harvey, I.C. 1981. An association of Lolium endophyte with ryegrass staggers. New Zealand Vet. J. 29, 185-186.

Florea, S., Phillips, T.D., Panaccione, D.G., Farman, M.L., Schardl, C.L. 2016. Chromosome-end knockoff strategy to reshape alkaloid profiles of a fungal endophyte. G3-Genes Genom. Genet. 6, 2601-2610.

Gallagher, R.T., Hawkes, A.D., Stewart, J.M. 1985. Rapid determination of the neurotoxin lolitrem $B$ in perennial ryegrass by high performance liquid chromatography with fluorescence detection. J. Chromatogr. 321, 217-223.

Gibert, A., Volaire, F., Barre, P., Hazard, L. 2012. A fungal endophyte reinforces population adaptive differentiation in its host grass species. New Phytol. 194, 561-571.

Guérin, P., 1898. Sur la présence d'un champignon dans l'ivraie. J. Bot. 12, 230-238.

Hesse, U., Schoberlein, W., Wittenmayer, L., Förster, K., Warnstorff, K., Diepenbrock, W., Merbach, W. 2003. Effects of Neotyphodium endophytes on growth, reproduction and drought-stress tolerance of three Lolium perenne L. genotypes. Grass Forage Sci. 58, 407415.

Hettiarachchige, I.K., Ekanayake, P.N., Mann, R.C., Guthridge, K.M., Sawbridge, T.I., Spangenberg, G.C., Forster, J.W. 2015. Phylogenomics of asexual Epichloë fungal endophytes forming associations with perennial ryegrass. BMC Evol. Biol. 15, 72.

Hovermale, J.T., Craig, A.M. 2001. Correlation of ergovaline and lolitrem B levels in endophyteinfected perennial ryegrass (Lolium perenne). J. Vet. Diagnost. Invest. 13, 323-327.

Inda, L., Sanmartin, I., Buerki, S., Catalán, P. 2014. Mediterranean origin and MioceneHolocene Old World diversification of meadow fescues and ryegrasses (Festuca subgenus Schedonorus and Lolium). J. Biogeog. 41, 600-614.

Jensen, A.M.D., Roulund, N. 2004. Occurrence of Neotyphodium endophytes in permanent grassland with perennial ryegrass (Lolium perenne) in Denmark. Agric. Ecosyst. Environ. 104, 419-427.

Johnson, L.J., De Bonth, A.C.M., Briggs, L.R., Caradus, J.R., Finch, S.C., Fleetwood, D.J., Fletcher, L.R., Hume, D.E., Johnson, R.D., Popay, A.J., Tapper, B.A., Simpson, W.R., Voisey, C.R., Card, S.D. 2013. The exploitation of epichloae endophytes for agricultural benefit. Fungal Divers. 60, 171-188.

Kaur, J., Ekanayake, P.N., Tian, P., Spangenberg, G. 2015. Discovery and characterisation of novel asexual Epichloë endophytes from perennial ryegrass (Lolium perenne L.). Crop Pasture Sci. 66, 1058-1070. 
König, J., Fuchs, B., Krischke, M., Mueller, M.J., Krauss, J. 2018. Hide and seek - Infection rates and alkaloid concentrations of Epichloë festucae var. Iolii in Lolium perenne along a land use gradient in Germany. Grass Forage Sci. 73, 510-516.

Lane, G.A., Tapper, B.A., Davies, E., Hume, D.E., Latch, G.C.M., Barker, D.J., Easton, H.S., Rolston, M.P. 1997. Effect of growth conditions on alkaloid concentrations in perennial ryegrass naturally infected with endophyte, in Bacon, C.W., Hill, N.S. (Eds), Neotyphodium/Grass Interactions. Springer, Athens, GA, p 179-182.

Lane, G.A., Christensen, M.J., Miles, C.O. 2000. Coevolution of fungal endophytes with grasses: The significance of secondary metabolites, in: Bacon, C.W., White, J.F. (eds), Microbial Endophytes. Marcel Dekker, New York, p. 341-388

Leuchtmann, A., Schardl, C.L.,Siegel M.R. 1994. Sexual compatibility and taxonomy of a new species of Epichloë symbiotic with fine fescue grasses. Mycologia 86, 802-812.

Leuchtmann, A., Bacon, C.W., Schardl, C.L., White, J.F. Jr, Tadych, M. 2014. Nomenclatural realignment of Neotyphodium species with genus Epichloë. Mycology 106, 13-25.

Lewis, G.C., Ravel, C., Naffaa, W., Astier, C., Charmet, G. 1997. Occurrence of Acremonium endophytes in wild populations of Lolium spp. in European countries and a relationship between level of infection and climate in France. Ann. Appl. Biol. 130, 227-238.

Lugtenberg, B.J.J., Caradus, J.R., Johnson, L.J. 2016. Fungal endophytes for sustainable crop production. FEMS Microbiol. Ecol. 92, fiw194.

Meijer, G., Leuchtmann, A. 1999. Multistrain infections of the grass Brachypodium sylvaticum by its fungal endophyte Epichloë sylvatica. New Phytol. 141, 355-368.

Moate, P.J., Williams, S.R.O., Grainger, C., Hannah, M.C., Mapleson, D., Auldist, M.J., Greenwood, J.S., Popay, A.J., Hume, D.E., Mace, W.J., Wales, W.J. 2012. Effects of wild-type, AR1 and AR37 endophyte-infected perennial ryegrass on dairy production in Victoria, Australia. Anim. Prod. Sci. 52, 1117-1130.

Moon, C.D., Craven, K.D., Leuchtmann, A., Clement, S.L., Schardl, C.L. 2004. Prevalence of interspecific hybrids amongst asexual fungal endophytes of grasses. Mol. Ecol. 13, 14551467.

Moon, C.D., Miles, C.O., Järlfors, U., Schardl, C.L. 2002. The evolutionary origins of three new Neotyphodium endophyte species from grasses indigenous to the Southern Hemisphere. Mycologia 94, 694-711.

Mulinti, P., Florea, S., Schardl, C.L., Panaccione, D.G. 2016. Modulation of ergot alkaloids in a grass-endophyte symbiosis by alteration of mRNA concentrations of an ergot alkaloid synthesis gene. J. Agric. Food Chem. 64, 4982-4989.

Oberhofer, M., Leuchtmann, A. 2012. Genetic diversity in epichloid endophytes of Hordelymus europaeus suggests repeated hosts jumpsand interspecific hybridizations. Mol. Ecol. 21, 2713-2726.

Oberhofer, M., Leuchtmann, A. 2014. Horizontal transmission, persistence and competition capabilities of Epichloë endophytes in Hordelymus europaeus grass hosts using dual endophyte inocula. Fungal Ecol. 11, 37-49.

Oliveira, J.A., Castro, V. 1998. Incidence of Neotyphodium endophytes in Spanish perennial ryegrass (Lolium perenne L.) accessions. Plant Genetic Res. Newslet. 113, 1-3.

Pennell, C.G.L., Popay, A.J., Rolston, M.P., Townsend, R.J., Lloyd-West, C.M., Card, S.D. 2016. Avanex unique endophyte technology: Reduced insect food source at airports. Environ. Entomol. 45, 101-108. 
Reed, K.F.M., Walsh, J.R., Cross, P.A., McFarlane, N.M., Sprague, M.A. 2004. Ryegrass endophyte (Neotyphodium lolii) alkaloids and mineral concentrations in perennial ryegrass (Lolium perenne) from southwest Victorian pasture. Aust. J. Exp. Agric. 44, 1185-1194.

Rodriguez, R.J., White, Jr J.F., Arnold, A.E., Redman, R.S. 2009. Fungal endophytes: diversity and functional roles. New Phytol. 182, 314-330.

Rowan, D.D., Dymock, J.J., Brimble, M.A. 1990. Effect of fungal metabolite peramine and analogs on feeding and development of Argentine stem weevil (Listronotus bonariensis). J. Chem. Ecol. 16, 1683-1695.

Saikkonen, K., Young, C.A., Helander, M., Schardl, C.L. 2016. Endophytic Epichloë species and their grass hosts: from evolution to applications. Plant Mol. Biol. 90, 665-675.

Schardl, C.L., Leuchtmann, A. 2005. The Epichloë endophytes of grasses and the symbiotic continuum, in Dighton, J., White, J.F., Oudemans, P. (eds), The fungal community. Its organization and role in the ecosystem. Taylor and Francis, Boca Raton, FL, USA, p 475-504.

Schardl, C.L., Leuchtmann, A., Tsai, H.F., Collet, M.A., Watt, D.M., Scott, D.B. 1994. Origin of a fungal symbiont of perennial ryegrass by interspecific hybridization of a mutualist with the ryegrass choke pathogen, Epichloë typhina. Genetics 136, 1307-1307.

Schardl, C.L., Young, C.A., Hesse, U., Amyotte, S.G., Andreeva, K., et al. 2013. Plant Symbiotic fungi as chemical engineers: multi genome analysis of the Clavicipitaceae reveals dynamics of alkaloid loci. PLOS Genet. 9(2), e1003323.

Schirrmann, M.K., Leuchtmann, A. 2015. The role of host-specificity in the reproductive isolation of Epichloe endophytes revealed by reciprocal infections. Fungal Ecol. 15, 29-38.

Shymanovich, T., Charlton, N.D., Musso, A.M., Scheerer, J., Cech, N.B., Faeth, S.H., Young, C.A. 2017. Interspecific and intraspecific hybrid Epichloë species symbiotic with the North American native grass Poa alsodes. Mycologia 109, 459-474.

Siegel, M., Bush, L. 1996. Defensive chemicals in grass-fungal endophyte associations. Recent Advan. Phytochem. 30:81-120.

Soto Barajas, M.C. 2016. Hongos endófitos Epichloë asociados a Lolium perenne : Identificación y producción de alcaloides. Ph.D. Thesis. Universidad de Salamanca.

Soto-Barajas, M.C., Zabalgogeazcoa, I., González-Martin, I., Vázquez-de-Aldana, B.R. (2018) Near-infrared spectroscopy allows detection and species identification of Epichloë endophytes in Lolium perenne. J Sci Food Agric 98, 5037-5044.

Sun, S., Clarke, B.B., Funk, C.R. 1990. Effect of fertilizer and fungicide applications on choke expression and endophyte transmission in chewings fescue, in Quisenberry, S.S., Joost, R.E. (eds), Proceedings of the international symposium on Acremonium/grass interactions. Baton Rouge, LA, p 62-66.

Tadych, M., Bergen, M.S., White, J.F. 2014. Epichloë spp. associated with grasses: new insights on life cycles, dissemination and evolution. Mycologia 106: 181-201.

Tamura, K., Stecher, G., Peterson, D., Filipski, A., Kumar, S. 2013. MEGA6: Molecular Evolutionary Genetics Analysis version 6.0. Mol. Biol. Evol. 30, 2725-2729.

Vázquez-de-Aldana, B.R., Zabalgogeazcoa, I., Rubio de Casas, R., García Ciudad, A., García Criado, B. 2010. Relationships between the genetic distance of Epichloë festucae isolates and the ergovaline and peramine content of their Festuca rubra hosts. Ann. Appl. Biol. 156, 51-61. 
White, T.J., Bruns, T., Lee, S., Taylor, J. 1990. Amplificacition and direct sequencing of fungal rigosomal RNA genes for phylogenetics, in Innis, M.A. (ed), PCR Protocols: A guide to methods and applications. Academic Press, San Diego, CA, p 315-322.

Wille, P.A., Aeschbacher, R.A., Boller, T. 1999. Distribution of fungal endophyte genotypes in doubly infected host grasses. Plant J. 18, 349-358.

Young, C.A., Tapper, B.A., May, K., Moon, C.D., Schardl, C.L., Scott, B. 2009. Indole-diterpene biosynthetic capability of Epichloë endophytes as predicted by Itm gene analysis. Appl. Environ. Microbiol, 75, 2200-2211.

Young, C.A., Hume, D.E., McCulley R.L. 2013. Fungal endophytes of tall fescue and perennial ryegrass: Pasture friend or foe? J. Anim. Sci. 91, 2379-2394.

Yue, Q., Johnson-Cicalese, J., Gianfagna, T.J., Meyer, W.A. 2000. Alkaloid production and chinch bug resistance in endophyte-inoculated chewings and stron creeping red fescues. J. Chem. Ecol. 26, 279-293.

Zabalgogeazcoa, I., Bony, S. 2004. Neotyphodium Research and Application in Europe, in Roberts, C., West, C., Spiers, D. (eds), Neotyphodium in cool season grasses. Blackwell Publishing, lowa, p. 23-33.

Zabalgogeazcoa, I., Vazquez de Aldana, B.R., Garcia Criado, B., Garcia Ciudad, A. 1999. The infection of Festuca rubra by the fungal endophyte Epichloë festucae in Mediterranean permanent grasslands. Grass Forage Sci. 54, 91-95. 


\section{FIGURES}

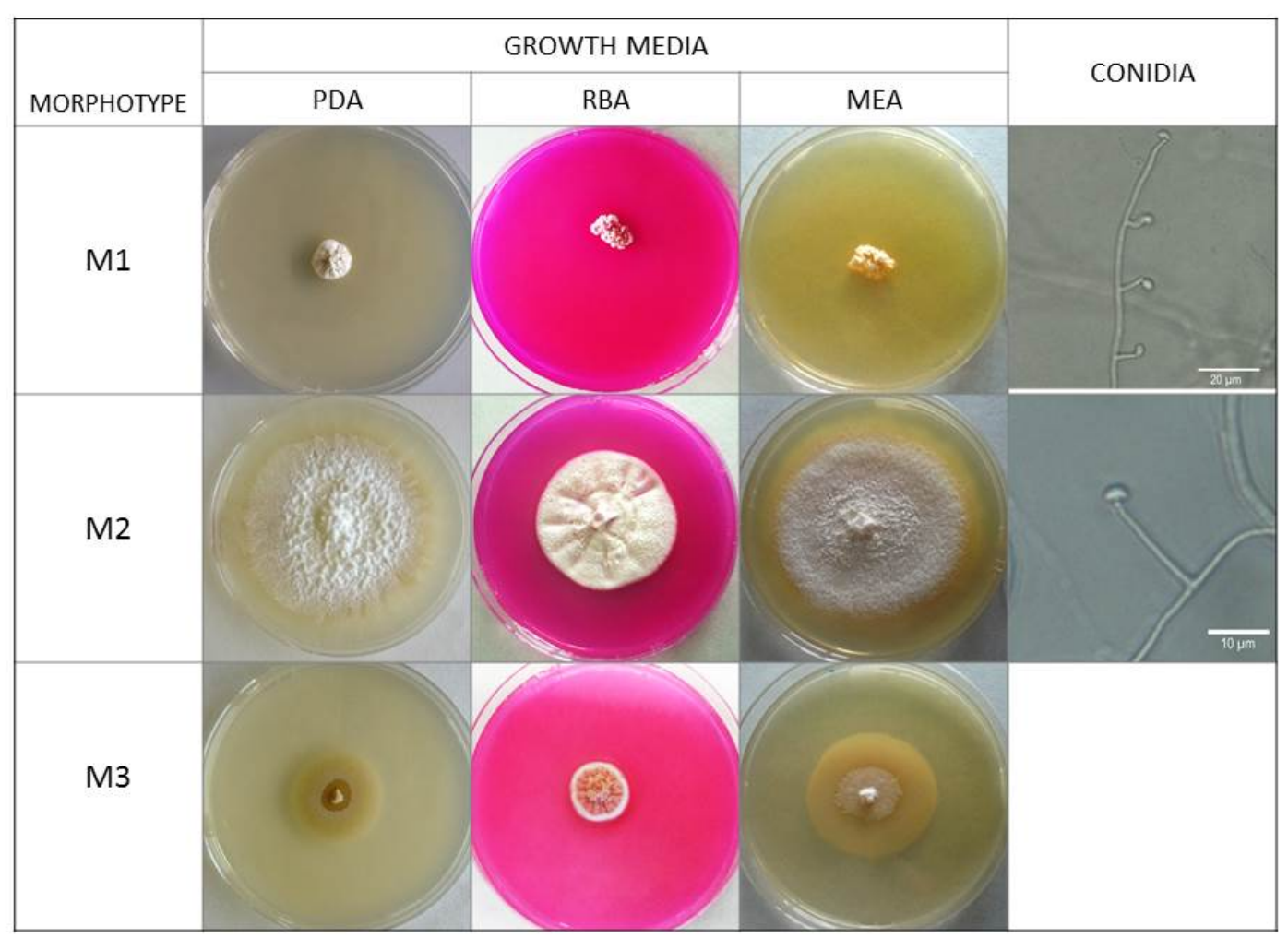

Figure 1. Morphotypes of Epichloë endophytes isolated from Lolium perenne plants in three growth media: potato dextrose agar (PDA), rose Bengal chloramphenicol agar (RBA), and malt extract agar (MEA). M1 morphotypes had mycelium of slow growth and a convoluted surface; M2 morphotypes had a faster growth rate and white cottony aerial mycelium; M3 morphotypes showed an intermediate growth rate and tan, smooth and flat mycelium. Conidia and conidiophores of M1 morphotype cultures grown in water agar at $10{ }^{\circ} \mathrm{C}$ and of $\mathrm{M} 2$ morphotype in PDA at room temperature $\left(\sim 22^{\circ} \mathrm{C}\right)$. M3 morphotype cultures did not sporulate. 
Figure 2. Maximum likelihood phylogeny based on nucleotide sequences of the ITS1-5.8S rDNA-ITS region of Epichloë endophytes of different morphotypes (M1, M2, M2S, M3) isolated from plants of Lolium perenne obtained at eight locations (LVA, LVE, VAF, CR, DIV, POR, TAB, $\mathrm{PI}$ ). The number after the dash indicates the number of strains from each location and morphotype with identical sequence. Genbank accession numbers are shown in parentheses. Bootstrap values are based on 1000 replicates. 


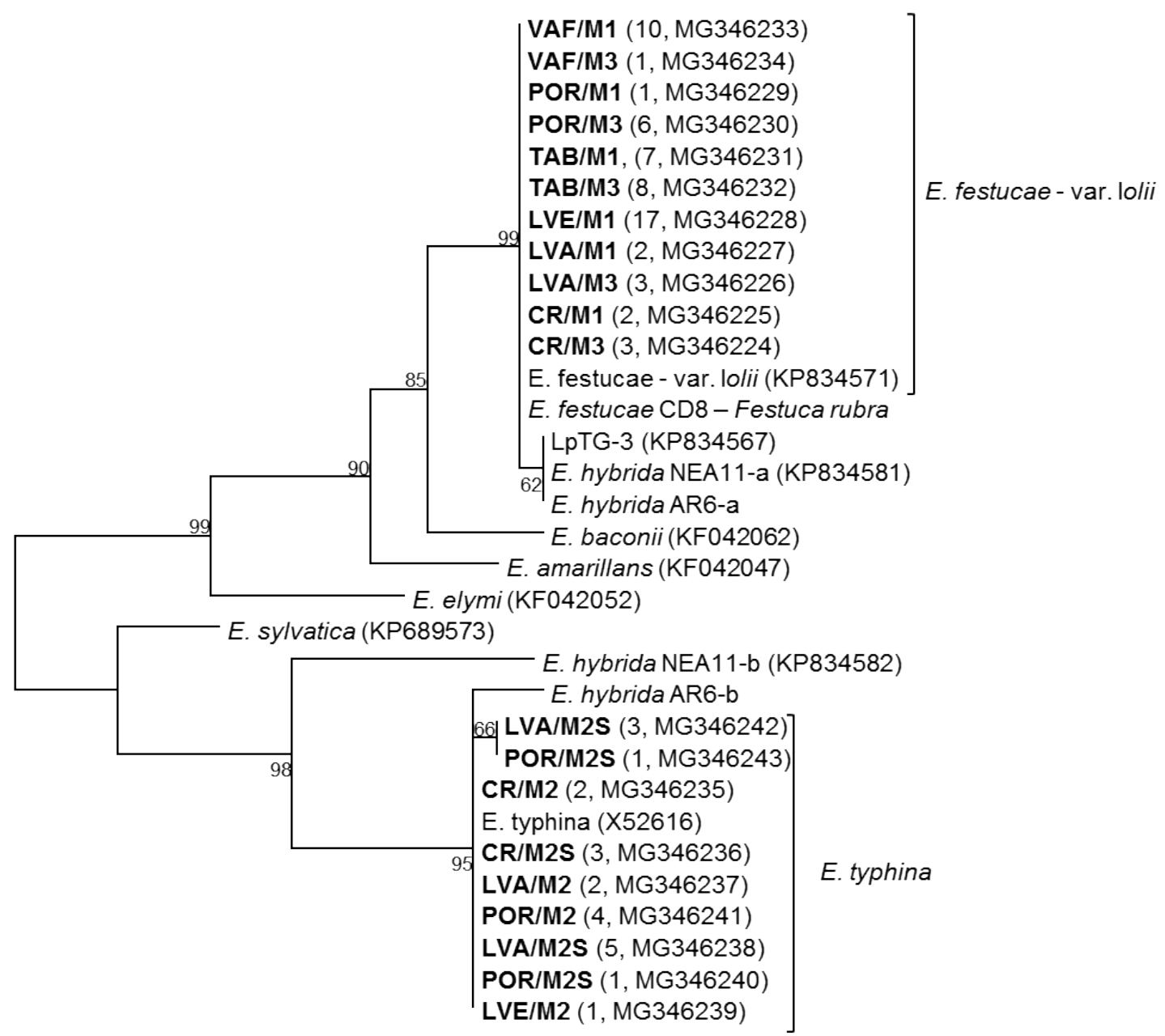

Figure 3. Maximum likelihood phylogeny based on nucleotide sequences of the tubB gene of Epichloë endophytes isolated from plants of Lolium perenne obtained at six locations: CR, LVA, LVE, TAB. POR and VAF. Sequences are grouped according to their morphotype (M1, M2, M2S, M3) and location. The number of strains from each location and morphotype with identical sequence, and the Genbank accession number of a sequence representative of each group appears in parentheses. Bootstrap values are based on 1000 replicates. 
Figure 4. Maximum likelihood phylogeny based on partial sequences of the tubB gene of Epichloë typhina strains obtained from different host plants, including four strains (bold) isolated from L. perenne plants in this study. Bootstrap values are based on 500 replicates. 
Figure 5. Representation of the plant sample coordinates on the factorial plane defined by components I and II of a principal component analysis. Samples are labelled according to their fungal morphotype (M1, M2, M3, M2S) with different symbols and colors. 


\section{TABLES}

Table 1. Sampling locations, prevalence of Epichloë endophytes in asymptomatic plants of Lolium perenne, and distribution of fungal morphotypes at each location.

\begin{tabular}{|c|c|c|c|c|c|c|c|c|c|}
\hline \multirow{2}{*}{ Location } & \multirow{2}{*}{$\begin{array}{l}\text { Type of } \\
\text { habitat }\end{array}$} & \multirow{2}{*}{ Coordinates } & \multirow{2}{*}{$\begin{array}{l}\text { Altitude } \\
\text { (m.a.s.I.) }\end{array}$} & \multirow{2}{*}{$\begin{array}{l}\text { Annual } \\
\text { precipitation } \\
(\mathrm{mm})\end{array}$} & \multirow{2}{*}{$\begin{array}{l}\text { Plants } \\
\text { collected } \\
\text { (n) }\end{array}$} & \multirow{2}{*}{$\begin{array}{c}\text { Number of } \\
\text { plants infected } \\
\text { (prevalence \%) }\end{array}$} & \multicolumn{3}{|c|}{ Number of strains of each fungal morphotype (\%) } \\
\hline & & & & & & & M1 & M2 & M3 \\
\hline Ciudad Rodrigo (CR) & Riverbank & $40.5800,-6.5161$ & 625 & 531 & 22 & $12(54.6)$ & $3(23.1)$ & $3(23.1)^{*}$ & $7(53.8)^{*}$ \\
\hline Divar (DIV) & Oak grassland & $40.7354,-5.7496$ & 817 & 521 & 32 & $11(34.4)$ & $3(27.3)$ & $2(18.2)$ & $6(54.5)$ \\
\hline Los Valles (LVA) & Oak grassland & $40.9389,-6.1267$ & 813 & 531 & 41 & $19(46.3)$ & $11(42.3)^{*}$ & $9(34.6)^{*}$ & $6(23.1)^{*}$ \\
\hline La Vecilla (LVE) & Rural path & $42.7065,-5.3867$ & 879 & 556 & 49 & $21(42.9)$ & $21(95.4)^{*}$ & $1(4.5)^{*}$ & $0(0.00)$ \\
\hline Porqueriza (POR) & Oak grassland & $40.9752,-5.9591$ & 807 & 531 & 48 & $14(29.2)$ & $2(14.3)$ & $2(14.3)$ & $10(71.4)$ \\
\hline Potes (PI) & Mountain pasture & 43.1467, -4.4734 & 1355 & 780 & 48 & $21(43.7)$ & $7(33.3)$ & $0(0.00)$ & $14(66.7)$ \\
\hline Tábara (TAB) & Oak grassland & $41.8391,-5.9785$ & 766 & 379 & 51 & $25(49.0)$ & $11(42.3)$ & $2(7.7)^{*}$ & $13(50.0)^{*}$ \\
\hline \multirow[t]{2}{*}{ Valle Fuentes (VAF) } & Shrubland & $42.9417,-5.2386$ & 1133 & 556 & 53 & $17(32.1)$ & $13(76.5)$ & $1(5.9)$ & $3(17.6)$ \\
\hline & & & & & $\Sigma=344$ & $\Sigma=140$ & $\Sigma=71$ & $\Sigma=20$ & $\Sigma=59$ \\
\hline
\end{tabular}

*Dual infections: CR: one plant infected by M2+M3; LVA: five plants infected by M2+M1, two plants with M2+M3; LVE: one plant with M1+M2; TAB: one plant with M2+M3 dual infection. 
Table 2. Alkaloid concentration and proportion of Lolium perenne plants containing them according to the morphotype of the Epichloë endophyte.

\begin{tabular}{|c|c|c|c|c|c|c|c|}
\hline \multirow{2}{*}{$\begin{array}{c}\text { Morphotype } \\
\text { Single infections }\end{array}$} & \multirow[b]{2}{*}{$\begin{array}{l}\text { Total number of } \\
\text { samples analyzed }\end{array}$} & \multicolumn{2}{|c|}{ Peramine } & \multicolumn{2}{|c|}{ Lolitrem B } & \multicolumn{2}{|c|}{ Ergovaline } \\
\hline & & $\begin{array}{c}\text { Number of } \\
\text { samples with } \\
\text { peramine (\%) }\end{array}$ & $\begin{array}{c}\text { Mean } \pm \text { SE } \\
\left(\mathrm{mg} \mathrm{kg}^{-1}\right)\end{array}$ & $\begin{array}{c}\text { Number of } \\
\text { samples with } \\
\text { lolitrem B (\%) }\end{array}$ & $\begin{array}{c}\text { Mean } \pm \text { SE } \\
\left(\mathrm{mg} \mathrm{kg}^{-1}\right)\end{array}$ & $\begin{array}{c}\text { Number of } \\
\text { samples with } \\
\text { ergovaline (\%) }\end{array}$ & $\begin{array}{c}\text { Mean } \pm \text { SE } \\
\left(\mathrm{mg} \mathrm{kg}^{-1}\right)\end{array}$ \\
\hline M1 & 53 & $36(67.9)$ & $2.62 \pm 0.29 a$ & $33(62.3)$ & $0.70 \pm 0.09 a$ & $25(47.3)$ & $0.67 \pm 0.14 a$ \\
\hline M2 & 8 & $3(37.5)$ & $3.79 \pm 2.52 \mathrm{ab}$ & $4(50.0)$ & $0.44 \pm 0.19 a b$ & $5(62.6)$ & $0.67 \pm 0.41 a$ \\
\hline M2S & 9 & $7(77.8)$ & $13.63 \pm 2.83 c$ & $2(22.2)$ & $0.11 \pm 0.07 \mathrm{~b}$ & $0(0.00)$ & -- \\
\hline \multirow[t]{2}{*}{ M3 } & 26 & $22(84.6)$ & $5.29 \pm 0.66 \mathrm{~b}$ & $20(76.9)$ & $1.06 \pm 0.15 \mathrm{c}$ & $22(84.6)$ & $0.85 \pm 0.15 a$ \\
\hline & $\Sigma=96$ & $\Sigma=68(70.8)$ & & $\Sigma=59(61.6)$ & & $\Sigma=52(54.2)$ & \\
\hline \multicolumn{8}{|l|}{ Double infections } \\
\hline M2/M1 & 3 & 3 & $3.46 \pm 0.66$ & 3 & $0.56 \pm 0.06$ & 3 & $1.85 \pm 0.90$ \\
\hline M2/M3 & 2 & 2 & $20.35 \pm 2.80$ & 2 & $0.60 \pm 0.03$ & 0 & -- \\
\hline M2S/M1 & 2 & 2 & $18.61 \pm 0.74$ & 1 & 0.53 & 0 & -- \\
\hline M2S/M3 & 1 & 1 & 23.89 & 0 & -- & 0 & -- \\
\hline
\end{tabular}

For each column, mean concentrations with different letters are statistically different at $P<0.05$ ). 
Figure S1. Alignment of a section of the sequence of each of the two alleles (AR06_Ef, AR06_Et) of the tubB gene, and the PCR amplicon (AR06_tub2) of the AR6 strain of the hybrid $E$. festucae var. Iolii $x$ E. typhina. The nucleotide sequence chromatogram of the PCR amplicon obtained from the AR6 strain shows overlapping peaks (enlarged at the bottom) in the positions where both alleles differ. Those signals in the chromatogram indicate the hybrid condition of the strain. 
Figure S2. Maximum likelihood phylogeny based on ITS sequences of 86 isolates of Epichloë endophytes of different morphotypes (M1, M2, M3, M2S) obtained from wild plants of Lolium perenne, plus reference sequences. GenBank accession numbers are shown in parentheses. 
Figure S3. Maximum likelihood phylogeny based on partial tubB sequences of 80 strains of Epichloë endophytes of different morphotypes (M1, M2, M3, M2S) obtained from wild plants of Lolium perenne, plus reference sequences. GenBank accession numbers are shown in parentheses.

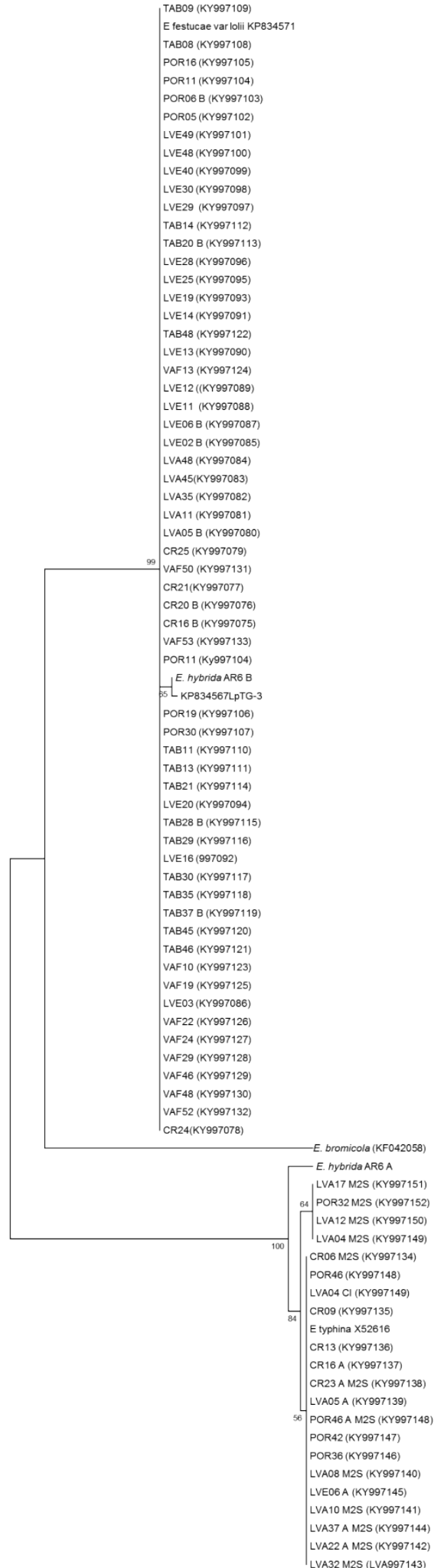

